\title{
MODELING THE HEAT TRANSFER PROCESS IN REFRIGERATION UNITS USED IN THE OIL INDUSTRY
}

\author{
Ibrahim Abulfaz Habibov ${ }^{l}$ \\ h.ibo@mail.ru \\ Rabiya Seydulla Nadjafkulieva ${ }^{l}$ \\ rabiya.nadjafkuliyeva@gmail.com \\ Sevinj Malik Abasova \\ seva-abasova@mail.ru \\ ${ }^{1}$ Department of "Engineering and computer graphics" \\ Azerbaijan State Oil and Industry University \\ 20 Azadliq ave., Baku, Azerbaijan, AZ 1010
}

\begin{abstract}
Currently, the main volume of associated petroleum gas production in Azerbaijan is carried out at offshore fields. About $30 \%$ of this volume is used for SOCAR's own technological needs of the oil company (to maintain the level of oil production). And the rest are transported to onshore facilities and further for processing to a gas processing plant. Subsequently, dry and purified gas is sent through a gas pipeline system for use by the population, or to a network of trunk pipelines for further export. When gas is transferred to the gas lift system and ashore, it is compressed in compressors, as a result of which the temperature of the working agent rises. The latter dramatically reduces the level of operational safety of the entire process. Based on the foregoing, ensuring the required gas quality at the outlet of the compressor unit is an important and urgent task.

Currently used in the gas cooling system, shell-and-tube type refrigeration units have several advantages: high heat transfer coefficient, operability in low and high pressure modes, simplicity of design and service. Along with this, these units are distinguished by large dimensions and high metal consumption, noise effect, vibration, etc.

A new design of a heat exchanger for cooling associated petroleum gas at the outlet of gas motor compressors is proposed.

Based on the modeling of the heat transfer process, mathematical models have been obtained that make it possible to adequately evaluate the cooling process in the proposed refrigeration device.

Keywords: gas engine compressors, compressor station, heat exchanger, refrigerator, working agent, seawater, compressed and heated gas.
\end{abstract}

DOI: $10.21303 / 2461-4262.2019 .001056$

\section{Introduction}

The oil and gas sector is a determining factor in the fuel and energy complex of Azerbaijan. Currently, the main volume of oil and gas production in the country is carried out at offshore fields, where the gas lift method is successfully used in parallel with the fountain method of oil production. Gas lift refers to a mechanized method of oil production, the principle of which is based on the ability of a gas to reduce the density of a liquid and to facilitate its ascent through tubing to the surface. In this case, the gas supply can be carried out using compressors.

The main task in developing an oil and gas or gas condensate field is both the creation of a rational technological system for the "production, collection, preparation and transportation" of produced products, and the use of highly reliable oil and gas production equipment with its effective location.

Currently, in the offshore fields of Azerbaijan ("Bahar", "Guneshli", "Sangachalymore-Duvanny-more-Bullf", "Bulla-sea", "Oil stones", "Mud Sopka", "Alaty", "Govsany", "Zyh", "Gumdeniz") a gas-lift method of production is used to maintain pressure at production wells. At the same time, associated petroleum gas (APG) is used as a working agent. Due to the lack of a sufficient number of appropriate compressor equipment, the degree of APG use in some offshore fields is not more than $80 \%$. 
Based on the foregoing, one of the main tasks assigned to the oil sector is providing a high-quality oilfield equipment for the "gas production, collection, preparation and transportation of gas" system and proper equipment placement [1-4].

About $70 \%$ of the total volume of gas produced from offshore fields is transferred to onshore facilities and then for processing to a gas processing plant, after which dry and purified gas is sent to the population and main gas pipelines for further transportation. The remaining part of the produced gas (about $30 \%$ ) is used for own technological needs by the oil company of the Republic of Azerbaijan (SOCAR).

The pressure required for this purpose is created by two and three stage compressors $[5,6]$.

At compressor stations (CS), gas engine compressors (GECs) are mainly used for gas compression. At the first GEC stage, the gas pressure rises to $P_{c}=1.8 \ldots 2.0 \mathrm{MPa}$. In this case, the temperature indicator in the compressed gas rises to $T_{g}=115 \div 118{ }^{\circ} \mathrm{C}$. For further use of the gas, before transferring it to the input of the 2 nd stage, it is necessary to cool it to a temperature $T=35 \div 38{ }^{\circ} \mathrm{C}$. For this purpose, a cooling system is provided in the compressor station, where the main elements of the system are refrigeration units.

Currently, shell-and-tube type refrigerators are used in the petrochemical, oil refining, and oil and gas producing fields of the industry, including SOCAR [9].

Although they are distinguished by a number of positive qualities (high heat transfer coefficient, operability in low and high pressure modes, simplicity of design and established service), but because of their large volume and metal consumption, increased noise during operation and vibration, as well as the complexity of the repair process they are used with certain restrictions $[10,11]$.

A new design of a refrigeration unit for cooling associated petroleum gas at the GEC outlet is developed and tested. A distinctive feature of the proposed refrigeration unit is that in it, in place of the bundles of heat exchange pipes inside the casing, a pipe with an external screw fin is installed.

In this regard, the article is devoted to modeling the heat transfer process in the proposed refrigeration device and the obtained mathematical models for determining the outlet temperatures, which allows an adequate assessment of the gas cooling process.

\section{Materials and methods}

Experimental studies were carried out in Oil and Gas Production Department named after Narimanov, where gas-powered two-stage piston compressors of the 10GKNAM 2/5-55 type are installed. In the process of gas compression at the outlet of the first stage of the GEC compressor cylinder, the values of pressure and temperature correspond to: $P=1.7 \div 1.9 \mathrm{MPa}, T_{g}=115 \div 118{ }^{\circ} \mathrm{C}$. Gas in this state can't be directed to subsequent stages of use; it must be cooled. To this end, the gas is re-routed to the cooling system.

The principle of operation of the technological cooling scheme is shown in Fig. 1.

As can be seen from Fig. 1 initially, seawater, when passing through filters (1), is cleaned of sand, algae and other fractions. Then it enters the separator (2) in order to re-filter.

Further, deeply purified water from the separator enters the tanks (7). To ensure uninterrupted water supply, the tanks are connected in series in 2 rows with pipes with a diameter of $150 \mathrm{~mm}$.

The purified water enters the inlet pipe of the heat exchanger (6). Upon completion of the cooling process, the used water is removed from the refrigeration unit and poured back into the sea.

Associated gas passing from the production well through the separator (4), purifying the additional fractions contained therein (except gas), enters the first stage of the gas engine compressor (5). Meanwhile, the temperature and pressure of the gas are respectively measured and recorded using measuring instruments (8) and (9).

The gas cooled from $116-118^{\circ} \mathrm{C}$ to $36-85^{\circ} \mathrm{C}$, which passes through the maintenance unit (6), in accordance with the technological regulations:

a) fed to the output line;

b) for re-compression is transferred to the 2nd stage of the compressor.

Functional diagram of the proposed refrigeration device is shown in Fig. 2. 


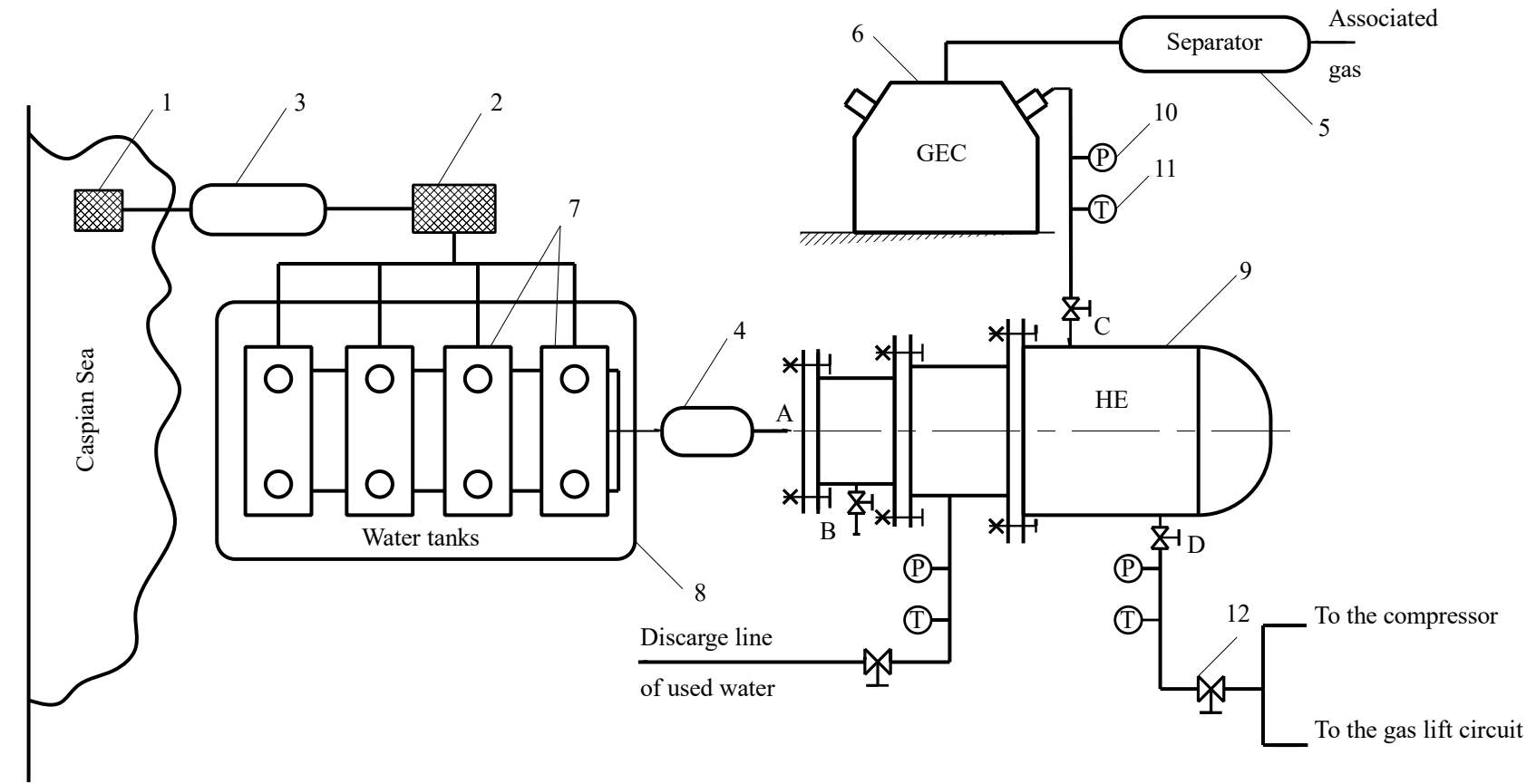

Fig. 1. The principle of operation of the technological scheme for cooling compressed gas in a gas lift system

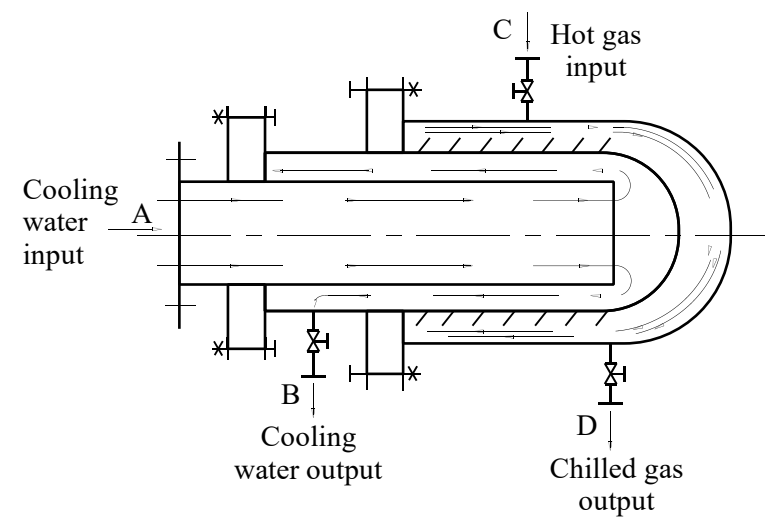

Fig. 2. Functional diagram of the refrigerator of a new design

As can be seen from the diagram, the cooling agent (sea water) entering the device through the input of the first pipe (A) is directed towards the hemispherical bottom of the second pipe. Then, filling the space between the first and second pipes, the water cools the heated gas moving between the second and third pipes, and moving away from the device through the output (B) is again supplied to the cooling system. In this case, the heated gas supplied to the device through the input (C), after cooling, is transmitted through the output (D) to the 2nd stage of the compressor or is sent to consumers through the main gas pipeline.

During experimental studies, in order to conduct comparative analyzes of changes in temperature indicators of the cooling and cooling agents at the inputs $\mathrm{A}$ and $\mathrm{C}$, as well as at the outputs $\mathrm{B}$ and D, thermometers WATTS TB-63\50 (for water) and TC7103AC (for gas) thermometers were installed.

The reliable operation of the refrigeration device depends on ensuring the final temperatures of both agents involved in the process under the given conditions.

To this end, the research task is simulation of the heat transfer process in the proposed refrigeration device and to obtain mathematical models that allow to estimate the final temperatures (cooling water and cooled gas). 


\section{Discussion of research results}

According to the functional diagram in the proposed refrigeration unit (Fig. 2), the heat exchange here is convection in nature, since heat is transferred here by circulation of the working agents of the liquid and gas. When describing the heat transfer process in refrigeration units, the following simplifications were made:

- given that during heat transfer, energy transfer occurs from the warmer part of the object to the less heated part, in our case, the highly heated gas located between the outer and middle pipes is cooled by transferring heat through the cold wall of the inner pipe;

- in the indicated section of the refrigerator, heat transfer occurs according to a descending regularity, therefore, the establishment of temperature in the final transition zone can be taken as simplified.

The heat exchange between the pipes in the cylindrical zone of the device can write a mathematical model of the process of heat distribution in the following form:

For a cooling agent (sea water) moving in a cylindrical zone of a device

$$
C_{w} \cdot \rho_{w} \cdot \frac{\delta T_{w}}{\delta t}=-v_{w} \cdot C_{w} \cdot \rho_{w} \cdot \frac{\partial T_{w}}{\partial x}+\propto\left(T_{g}-T_{w}\right) .
$$

For cooled agent (compressed gas)

$$
C_{g} \cdot \rho_{g} \cdot \frac{\partial T_{g}}{\partial t}=-v_{g} \cdot C_{g} \cdot \rho_{g} \frac{\partial T_{g}}{\partial x}-\propto\left(T_{g-} T_{w}\right)
$$

Here $C_{w}$ and $C_{g}$ are, respectively, the heat capacities of the cooling water and gas; $\rho_{w}$ and $\rho_{g}$ are, respectively, the density of cooling water and gas; $T_{w}$ and $T_{g}$ are, respectively, the temperature of the cooling water and gas;

$$
\alpha=\frac{2}{R} \cdot \alpha_{1}
$$

where $\alpha_{1}$ is the heat transfer coefficient and characterizes the intensity of the cooling process.

Let's suppose that for the system of equations (1), (2) the initial conditions are given

$$
\begin{aligned}
& T_{w}(x, o)=\gamma_{w}(x), \\
& T_{g}(x, o)=\gamma_{g}(x) .
\end{aligned}
$$

Since the temperature is known in the part where the cooled agent is introduced into the device, the boundary condition for equation (2) can be written as

$$
T_{g}(o, t)=f_{g}(t) \text {. }
$$

It is clear that for equation (1) the boundary condition, as can be seen from Fig. 1 should be defined at the beginning of the spherical structure of the device. To obtain this boundary condition, let's write the heat balance equation for the part where the spherical structure is located

$$
\pi R^{2} v_{w} \cdot C_{w} \cdot \rho_{w} \cdot\left(T_{w}(0, t)-T_{w}^{0}(t)\right)=2 \pi r 2 R \alpha_{1}\left(T_{g}(l, t)-T_{w}(0, t)\right)
$$

From here, it is possible to obtain the boundary condition at the beginning of the cylindrical pipe along which the cooling agent moves

$$
T_{w}(0, t)=\frac{4 r \alpha_{1} T_{g}(l, t)+R v_{w} \cdot C_{w} \cdot \rho_{w} T_{w}^{0}(t)}{R v_{w} \cdot C_{w} \cdot \rho_{w}+4 r \alpha_{1}} .
$$


Here $T_{w}^{0}(t)$ is the temperature in the part where the cooling agent is introduced into the device.

Thus, the problem is reduced to finding the functions $T_{w}(x, t)$ and $T_{g}(x, t)$ satisfying the system of equations (1), (2), the initial conditions (3), (4) and the boundary conditions (5), (6). But since it is impossible to analytically solve the problem in general, it becomes necessary to use numerical methods of solution.

To find a numerical solution to problem (1)-(6), let's use the finite difference method. For this purpose, let's introduce grids in the region $\{0 \leq x \leq l, 0 \leq t \leq T\}$

$$
\bar{\omega}_{h \tau}=\left\{\begin{array}{l}
\left(x_{i}, t_{j}\right): x_{i}=i h, t_{j}=j \tau, \\
i=0,1,2, \ldots, n, j=0,1,2, \ldots, m
\end{array}\right\} .
$$

Here $h=l / n, \quad \tau=\frac{T}{m}$ are the network steps for the variables $x$ and $t$, respectively. In the introduced grid $\bar{\omega}_{h \tau}$, let's write the finite-difference analogue of problem (1)-(6) in the following form:

$$
\begin{gathered}
C_{w} \cdot \rho_{w} \frac{T_{w_{i}}^{j}-T_{w}^{j-1}}{\tau}-\vartheta_{w} \cdot C_{w} \cdot \rho_{w} \frac{T_{w_{i}}^{j}-T_{w_{i}}^{j}}{h}+\alpha\left(T_{w_{i}}^{J-1}-T_{w_{i}}^{j-1}\right), \\
C_{g} \cdot \rho_{g} \cdot \frac{T_{g_{i}}^{j}-T_{g_{i}}^{j-1}}{\tau}=-\vartheta_{g} \cdot C_{g} \cdot P_{g} \frac{T_{g_{i}}^{j}-T_{g_{i}-1}^{j}}{\tau}-\propto\left(T_{g_{i}}^{j-1}-T_{g_{i}}^{j-1}\right), \\
i=\overline{1, n}, j=\overline{0, m,} \\
T_{w i}^{0}=\gamma_{w i}, \\
T_{g i}^{0}=\gamma_{g i}, \\
T_{w 0}^{j}=\frac{4 r \alpha_{1} T_{g n}^{j-1}+R v_{w} \cdot C_{w} \cdot \rho_{w} T_{w}^{0 j}}{R v_{w} \cdot C_{w} \cdot \rho_{w}+4 r \alpha_{1}} \\
T_{g 0}^{j}=f_{g}^{j} .
\end{gathered}
$$

As can be seen, the discrete analogue of the differential problem (1)-(6) is the system of linear algebraic equations (7)-(12). And the variables of this system are approximate values $T_{w_{i}}^{j}$ and $T_{g_{i}}^{j}$ at the nodal points of the grid $\bar{\omega}_{h \tau}$ of the desired functions $T_{w}(x, t)$ and $T_{g}(x, t)$. From this system, the values of the variables $T_{w_{i}}^{j}$ and $T_{g_{i}}^{j}$ can be found in the following formulas in explicit form:

$$
\begin{gathered}
T_{w_{i}}^{j}=\frac{h \cdot C_{w} \cdot \rho_{w}}{h \cdot C_{w} \cdot \rho_{w}+\tau \cdot \vartheta_{w} \cdot C_{w} \cdot \rho_{w}} \cdot T_{w_{i}}^{j-1}+\frac{\tau \cdot \vartheta_{w} \cdot C_{w} \cdot \rho_{w}}{h \cdot C_{w} \cdot \rho_{w}+\tau \cdot \vartheta_{W} \cdot C_{w} \cdot \rho_{w}} \cdot T_{w_{i-1}}^{j}+ \\
+\frac{\propto \cdot h \cdot \tau}{h \cdot C_{w} \cdot \rho_{w}+\tau \cdot \vartheta_{w} \cdot C_{w} \cdot \rho_{w}}\left(T_{g_{i}}^{j-1}-T_{w_{i}}^{j-1}\right) . \\
T_{g_{i}}^{j}=\frac{h \cdot C_{g} \cdot \rho_{g}}{h \cdot C_{g} \cdot \rho_{g}+\tau \vartheta_{g} \cdot C_{g} \cdot \rho_{g}} T_{g_{i}}^{j-1}+\frac{\tau \cdot \vartheta_{g} \cdot C_{g} \cdot \rho_{g}}{h \cdot C_{g} \cdot \rho_{g}+\tau \vartheta_{g} \cdot C_{g} \cdot \rho_{g}} T_{g_{i-1}}^{j}+ \\
+\frac{\propto \cdot h \cdot \tau}{h \cdot C_{g} \cdot \rho_{g}+\tau \cdot \vartheta_{g} \cdot C_{g} \cdot P_{g}} \cdot\left(T_{g_{i}}^{j-i}-T_{w_{i}}^{j-1}\right) .
\end{gathered}
$$

The results of a comparative analysis of the temperature values of the cooling agents obtained as a result of the calculations based on the proposed model and determined by experimental measurements at the output of the device are given in Table 1. 
Table 1

Comparative analysis of temperature indicators of the cooling agents

\begin{tabular}{cccccccc}
\hline Coolants & \multicolumn{7}{c}{ The flow rate of cooling water, m/s. } \\
\cline { 2 - 8 } & $\mathbf{0 . 5}$ & $\mathbf{1 . 0}$ & $\mathbf{1 . 5}$ & $\mathbf{2 . 0}$ & $\mathbf{2 . 5}$ & $\mathbf{3 . 0}$ \\
\hline Gas temperature, ${ }^{\circ} \mathrm{C}$ & $\underline{56.8^{*}}$ & $\underline{47.8}$ & $\underline{36.8}$ & $\underline{36.1}$ & $\underline{35.7}$ & $\underline{35.3}$ \\
Water temperature, ${ }^{\circ} \mathrm{C}$ & 39.5 & 34.3 & 27.7 & 25.3 & 23.7 & 23.4
\end{tabular}

Note: In the numerators of fractions, indicators obtained by calculations are indicated, and in the denominator, experimentally determined indicators.

\section{Conclusions}

Thus, as can be seen from the analysis of Table 1, with a change in the flow rate of the cooling agent within $\vartheta \quad 0.5-1.5 \mathrm{~m} / \mathrm{s}$, an intensive decrease in the temperature of the cooled gas occurs. However, a further increase in speed has little effect on the cooling process.

Mathematical models obtained on the basis of modeling the heat transfer process allow to adequately evaluate the cooling process in the proposed refrigeration device, and they can be used in other types of shell-and-tube refrigeration units.

\section{References}

[1] Asadov, O. S., Aliev, V. I., Makarov, V. V. (2011). Noviy nauchniy podhod k opredeleniyu real'noy proizvoditel'nosti porshnevyh kompressorov v sistemah gazlifta i transporta gaza. Moscow: «Sputnik +», 386.

[2] Krets, V. G., Rudachenko, A. V., Shmurygin, V. A. (2016). Mashiny i oborudovanie gazonefteprovodov. Sankt-Peterburg: Lan', 376.

[3] Kantyukov, R. A. (2014). Kompressory v tehnologicheskih protsessah: gazoraspredelitel'nye, kompressornye stantsii magistral'nyh gazoprovodov i avtomobil'nye gazonakopitel'nye kompressornye stantsii. Kazan': Kazan. nats. issled. tehnolog. un-t, 645 .

[4] Ong, C. W., Chen, C.-L. (2019). Technical and economic evaluation of seawater freezing desalination using liquefied natural gas. Energy, 181, 429-439. doi: https://doi.org/10.1016/j.energy.2019.05.193

[5] Mazyan, W., Ahmadi, A., Ahmed, H., Hoorfar, M. (2016). Market and technology assessment of natural gas processing: A review. Journal of Natural Gas Science and Engineering, 30, 487-514. doi: https://doi.org/10.1016/j.jngse.2016.02.010

[6] Bahadori, A. (2014). Liquefied Natural Gas (LNG). Natural Gas Processing, 591-632. doi: https://doi.org/10.1016/b978-0-08099971-5.00013-1

[7] Bulygin, Yu. A., Baranov, S. S. (2015). Teploobmennye apparaty v neftegazovoy promyshlennosti: kursovoe proektirovanie. Voronezh: FGBOU VPO «Voronezhskiy gosudarstvennyy tehnicheskiy universitet», 100.

[8] Jacobs, G. G., Liebenberg, L. (2016). The influence of timed coolant injection on compressor efficiency. Sustainable Energy Technologies and Assessments, 18, 175-189. doi: https://doi.org/10.1016/j.seta.2016.10.010

[9] Hou, M., Wu, Z., Hu, J., Zhang, L., Luo, E. (2019). Experimental study on a thermoacoustic combined cooling and power technology for natural gas liquefaction. Energy Procedia, 158, 2284-2289. doi: https://doi.org/10.1016/j.egypro.2019.01.251

[10] Energy and Exergy Analyses of Natural Gas Liquefaction Cycles (2014). Handbook of Liquefied Natural Gas, $185-228$. doi: https://doi.org/10.1016/b978-0-12-404585-9.00004-0

[11] Guo, B., Liu, X., Tan, X. (2017). Gas Lift. Petroleum Production Engineering, 549-601. doi: https://doi.org/10.1016/b978-012-809374-0.00017-9 